\title{
Characterization of adenomas and metastases in the adrenals: comparison between spin-echo sequences and Gd-DTPA enhanced dynamic studies
}

\author{
Ettore Squillaci ${ }^{1}$, Antonello Vidiri ${ }^{2}$, Marcello Crecco $^{2}$, Federico Maspes ${ }^{1}$, Ezio Fanucci ${ }^{1}$, Maria Luisa Grandinetti ${ }^{2}$, \\ and Giovanni Simonetti ${ }^{1}$
}

${ }^{1}$ Istituto di Radiologia, Universita' di Roma “Tor Vergata”, Ospedale S. Eugenio, P. le Umanesimo 10, I-0144 Rome, Italy

${ }^{2}$ Radiologia e Diagnostica per Immagini, Istituto Regina Elena Rome, Italy

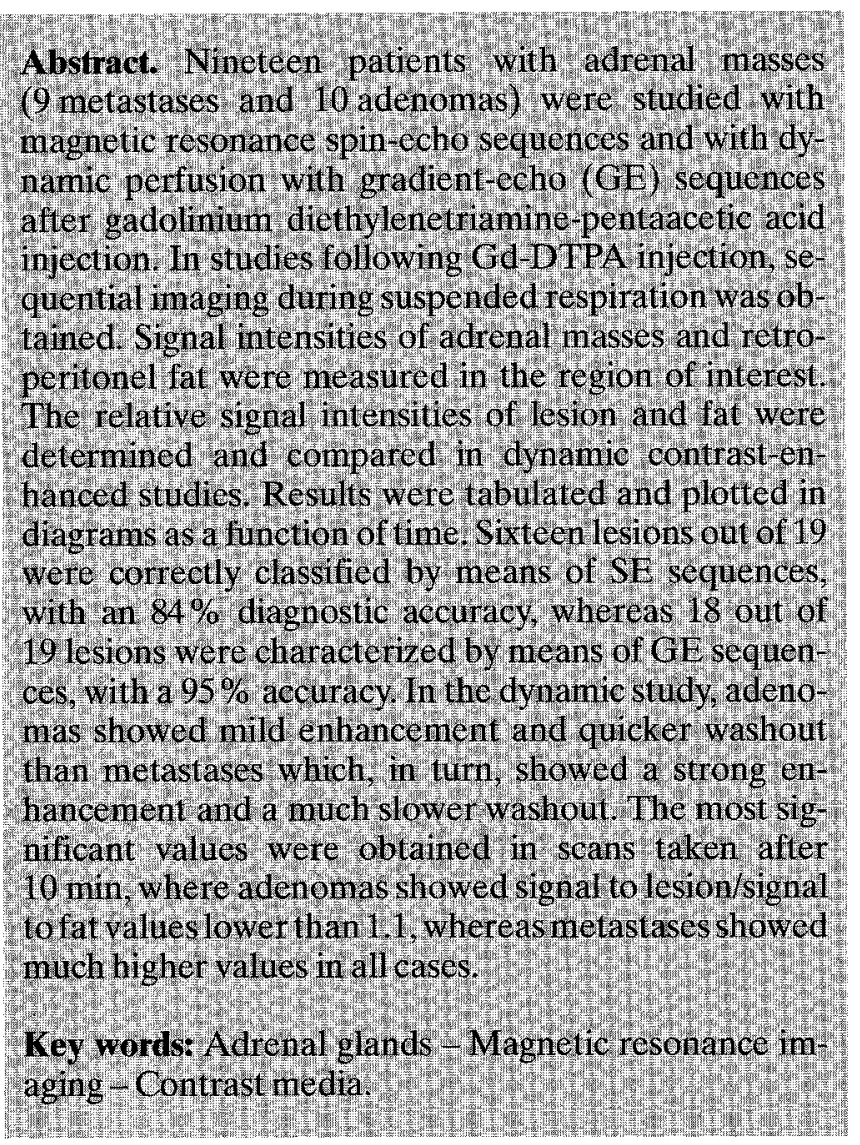

\section{Introduction}

Adrenal lesions are incidentally observed on computerized tomography (CT) in around $1 \%$ of cases, whereas those found at autopsy in $2-10 \%[1,2]$. It is therefore of great importance to characterize such lesions in oncologic patients, where the presence of an adenoma or of

Correspondence to: E. Squillaci adrenal metastases may affect the choice of therapeutic modality and prognosis.

$\mathrm{CT}$ is used to discriminate benign from malignant lesions. Besides morphologic criteria, attenuation values are also used. According to several authors, lesions with attenuation values below zero are almost always benign, whereas those with values above $15 \mathrm{HU}$ were mostly malignant $[2,3]$. Such findings, though, are not always in agreement; therefore, though CT sensitivity is very high in recognizing such lesions, its specificity is low [2-4].

In recent years, several studies have proposed the use of magnetic resonance (MR), to characterize adrenal masses, based on the signal intensity of spin-echo (SE) T1and T2-weighted sequences, using both a low and high field intensity magnet, with different criteria to assess results [5-9]. Studies have also proposed the use of gradientecho (GE) sequences and intravenous (i.v.) administration of paramagnetic contrast media, allowing dynamic measurement of the enhancement pattern of both benign and malignant lesions [10-12].

In our prospective study results obtained with SE sequences were compared with those of GE sequences with dynamic study after i. v. injection of paramagnetic contrast media, in order to characterize adrenal adenomas and metastases.

\section{Methods}

Nineteen patients with adrenal pathology (10 adenomas and 9 metastases) were studied prospectively with MR. Sixteen patients had known malignant disease demonstrated in other organs ( 7 in the pelvis, 5 in the breast, 3 in the rectum and 1 in the stomach). All diagnoses were histologically proven, either at surgery $(n=3)$, CT-guided biopsy $(n=9)$ or follow-up $(n=7)$. The mean diameter was $2.2 \mathrm{~cm}$ for adenomas and $3.6 \mathrm{~cm}$ metastases.

No patient had bilateral lesions. In the seven cases who underwent follow-up, there were two metastases and five adenomas. The minimum period of follow-up was 6 months (range $=6-11$ months). Follow-up was per- 


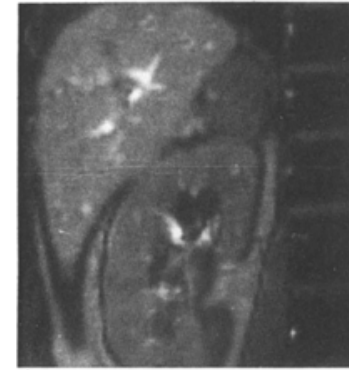

$1 \mathbf{a}$

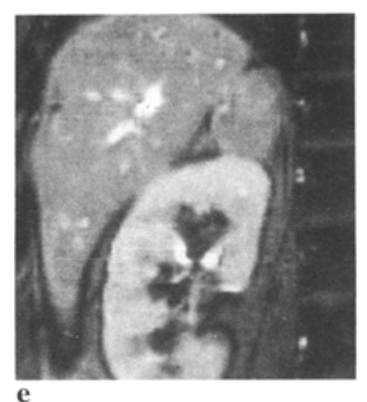

e

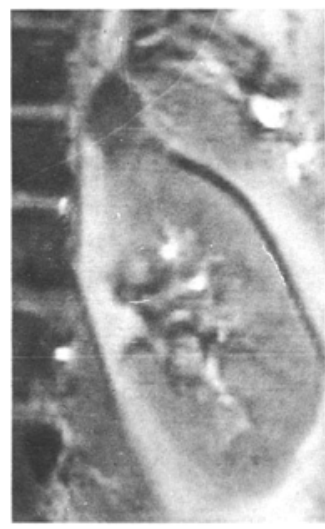

2a

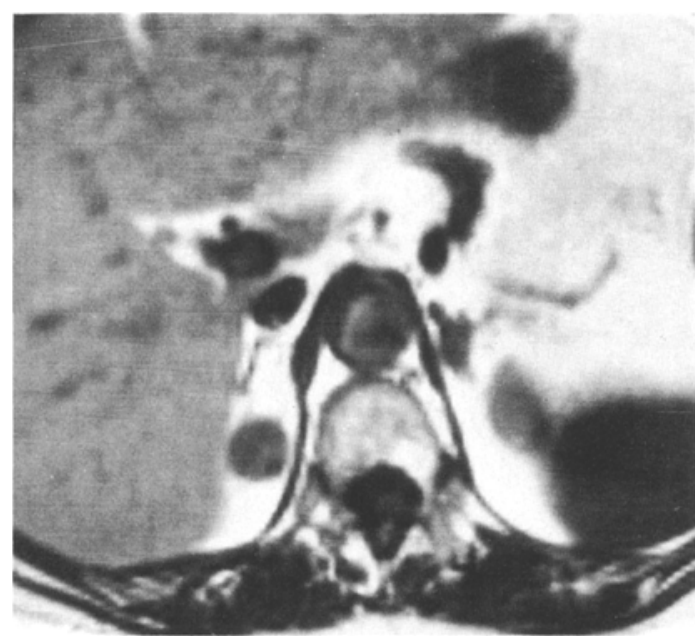

$3 \mathbf{a}$

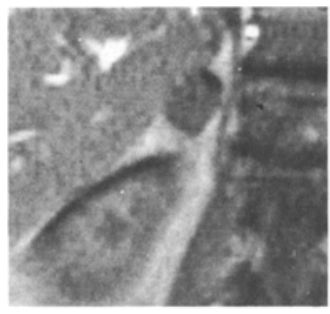

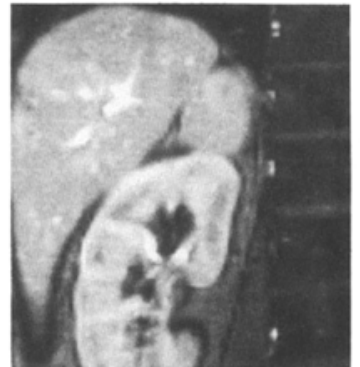

b

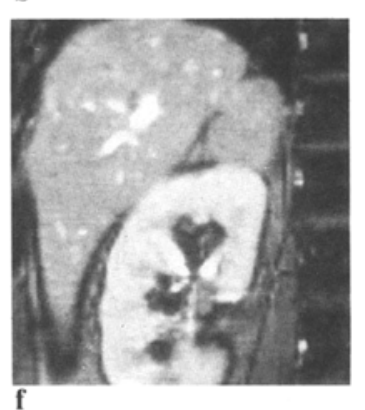

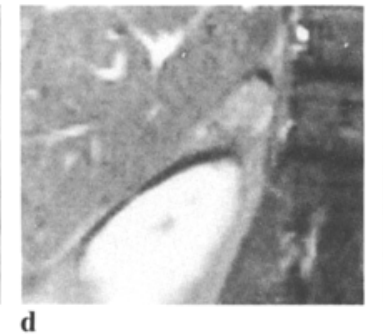

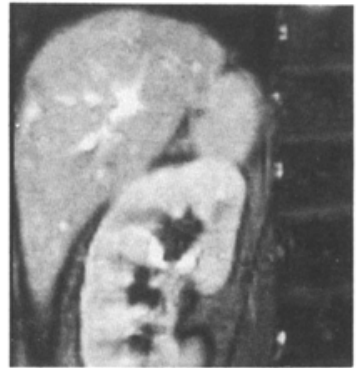

c

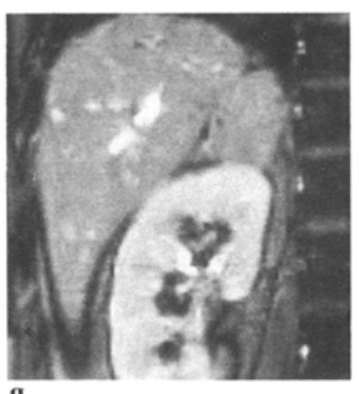

g

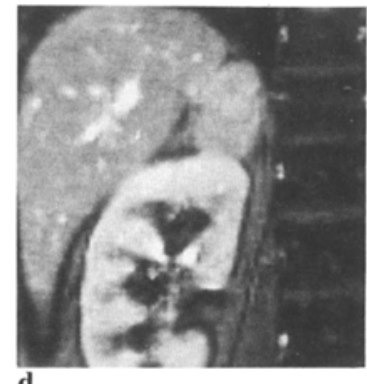

d

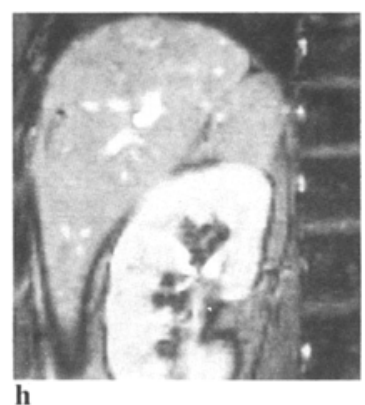

h
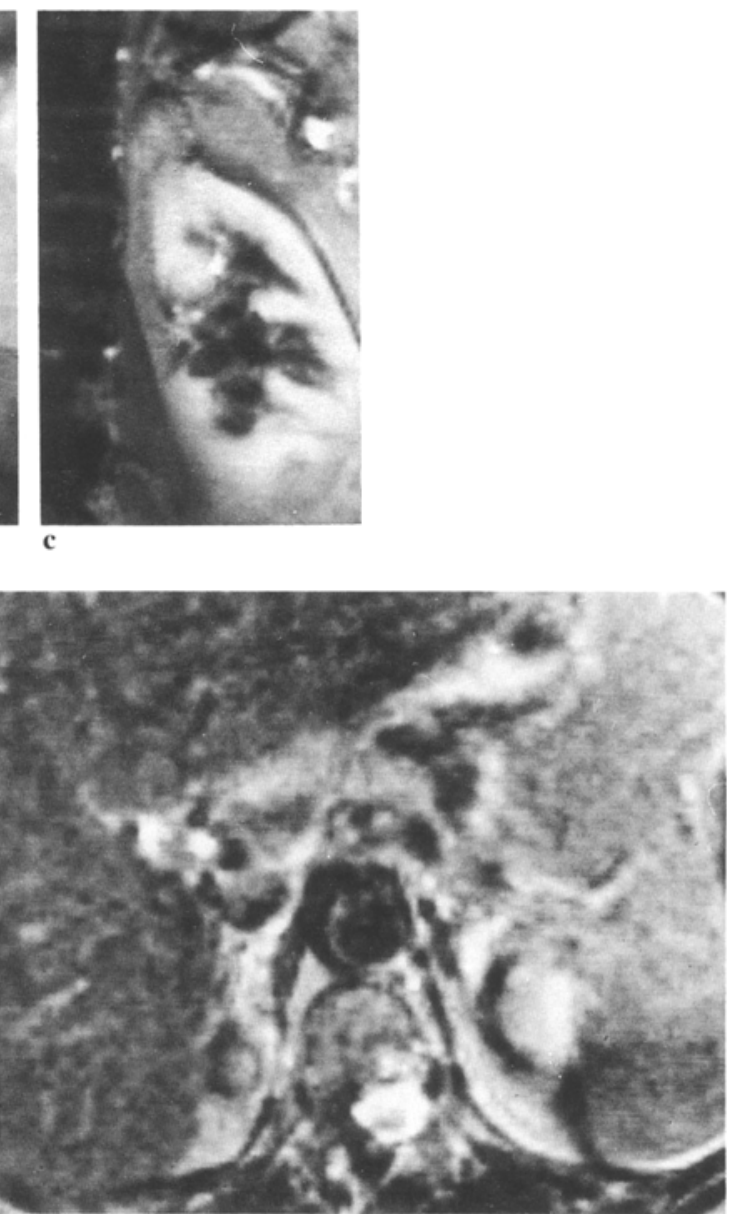

b
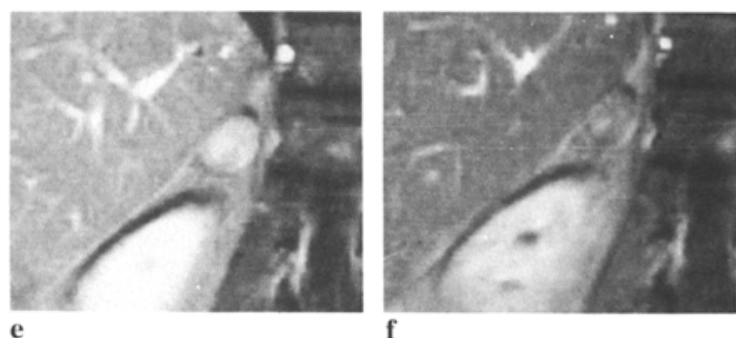
formed both clinically and by US $(n=1), \mathrm{CT}(n=5)$, MR $(n=1)$. All patients had undergone CT exam before MR examination.

All MR images were obtained using a 1.5-T superconductive magnet (Siemens Magnetom) and a body coil. Preliminary scans were performed using T1- and T2weighted SE sequences; dynamic scans were then performed on the lesion plan by means of coronal GE sequences, both before and after i.v. injection of $0.1 \mathrm{mmol} / \mathrm{kg}$ of Gadolinium diethylenetriaminepentaacetic acid (Gd-DTPA; Schering, Berlin). SE T1weighted sequences were obtained with a $500 \mathrm{~ms}$ repetition time (TR) and a 15-30 ms echo time (TE) with two or four excitations; T2-weighted sequences with a TR of $2500 \mathrm{~ms}$ and a TE of 30 and $90 \mathrm{~ms}$ and 1 excitation. Slices of $5 \mathrm{~mm}$ thickness were used with a $2 \mathrm{~mm}$ gap and imaged using a $256 \times 256$ matrix. In all SE sequences, a pre-saturation program was used to reduce respiratory or peristaltic artifacts.

GE sequences were obtained during suspended respiration with an acquisition time of about $17 \mathrm{~s}$. The flip angle used was $70^{\circ}$ with a TR of $50 \mathrm{~ms}$ and a TE of $12 \mathrm{~ms}$.

$\mathrm{SE}$ sequences were performed in axial and coronal planes to better demonstrate the lesion and its size, whereas GE sequences were mainly performed in the coronal plane to reduce respiratory and peristaltic motion artifacts.

The dynamic study was performed with GE sequences on a plane pre-selected on the previous SE study where the lesion diameter was greater. Gd-DTPA was injected at a dose of $0.1 \mathrm{mmol} / \mathrm{kg}$, followed by $50 \mathrm{cc}$ saline, and consecutive GE scans were performed 1, 2, 3, 4, 5, 6, 8, 10, 12, and $15 \mathrm{~min}$ after the Gd-DTPA injection.

For the assessment of the dynamic study the mean signal intensities, both inside the adrenal mass and the subcutaneous fat were recorded. Inside the lesion, the greatest possible region of interest, (ROI), compatible with the lesion to be measured was used, avoiding borderline areas to minimize the influence of the partial-volume effect. To evaluate the signal intensity of fat, the size of the ROI measurement was always $1.5 \mathrm{~cm}$.

The signal intensity ratio between the lesion and retroperitoneal fat (SL/SF) was calculated in all sequences ob-

Fig. 1a-h. Metastasis of the right adrenal. MR GE sequences a preand post-injection of Gd-DTPA, images obtained at $\mathbf{b} 1 \mathrm{~min}$, c $2 \mathrm{~min}, \mathbf{d} 4 \mathrm{~min}$, e $6 \mathrm{~min}, \mathbf{f} 8 \mathrm{~min}, \mathrm{~g} 10 \mathrm{~min}, \mathbf{h} 15 \mathrm{~min}$. There is quick enhancement of the lesion in the sequence obtained after $1 \mathrm{~min}$. Enhancement is also relatively high in the sequences obtained after 10 and $15 \mathrm{~min}$

Fig. 2 a-c. Adenoma of the left adrenal. MR GE sequence a pre and b-c-post Gd-DTPA. Typical low signal lesion before contrast medium injection with a moderate enhancement after $2 \min \mathbf{b}$ and a quick washout after 5 min $\mathbf{c}$ from the contrast medium injection

Fig.3a-f. Adenoma of the right adrenal. MR SE a T1 and b T2 weighted images show a low signal lesion on the T1 image, and a moderate high signal lesion on the $\mathrm{T} 2$ image. The signal intensity of the lesion does not allow correct characterization. MR GE sequences $\mathbf{c}$ pre- and $\mathbf{d}-\mathbf{f}$ post Gd-DTPA injection shows a moderate enhancement after $1 \mathrm{~min} \mathbf{d}$ with a quick washout after $3 \mathbf{e}$ and $10 \mathbf{f ~ m i n}$, typical of benign lesions
Table 1. Relative signal intensity (SL/SF) of adrenal adenomas ( $\Delta$ ) and metastases ( $\square$ ) on GE sequences with Gd-DTPA in dynamic study

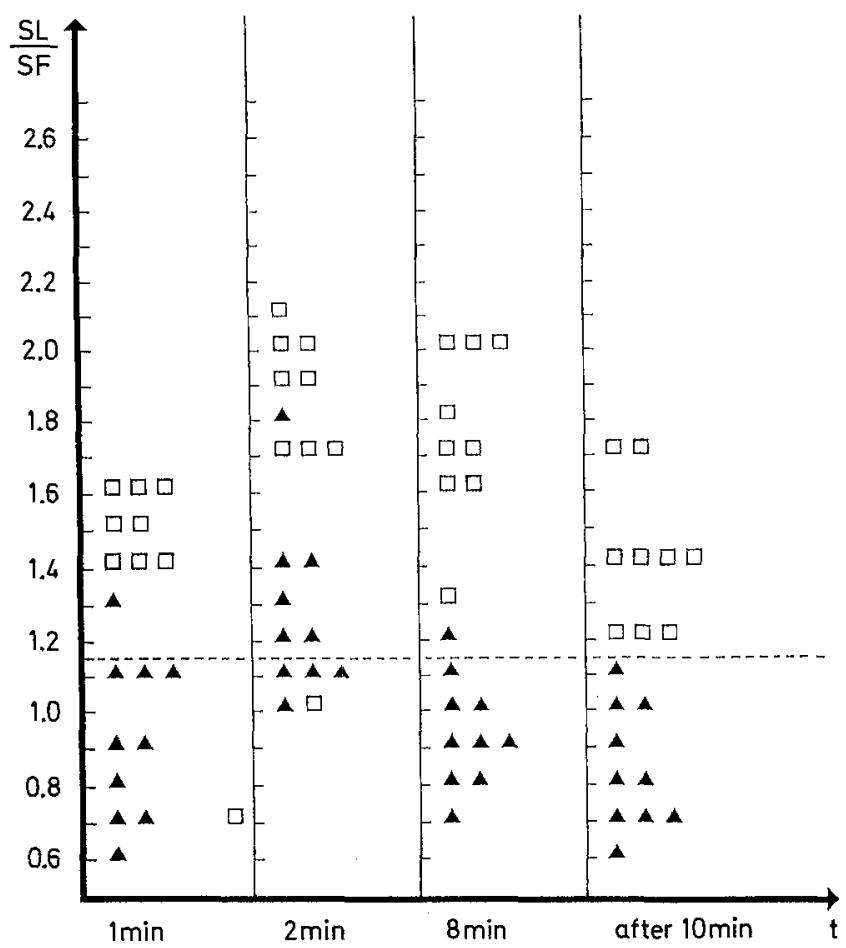

tained. Results were tabulated and plotted in diagrams as a function of time.

\section{Results}

All adenomas gave a low signal intensity on T1-weighted images when using an SE sequence, while on T2-weighted sequences, signal intensity was low in eight cases and slightly hyperintense in two cases. Metastases appeared hypointense on SE T1- and hyperintense on SE T2weighted sequences in all cases.

By means of the GE sequences, signal intensities from lesions were recorded before and after Gd-DTPA injection.

In scans performed in baseline conditions, metastases showed SL/SF ratios between 0.6 and 0.9 , whereas adenomas showed values between 0.3 and 0.6 . In one patient alone was the SL/SF ratio for adenoma in the range found for metastatic lesions. Following Gd-DTPA administration, metastases behaved substantially differently from adenomas, showing a quick and progressive enhancement with peak values between 1.7 and 2.1, after 2-3 min. Enhancement remained high in later scans, reaching values above 1.1 after $10 \mathrm{~min}$ (Fig.1). Only a metastasin from a rectal carcinoma showed abnormal SL/SF values $(0.7$, 1 min after Gd-DTPA injection and 1.0, 2 min after), which were similar to that of benign lesions though in scans obtained 8 and 10 min later, the lesion showed higher values than adenomas.

Following Gd-DTPA injection, adenomas showed a moderate enhancement with a peak after about $2 \mathrm{~min}$ 


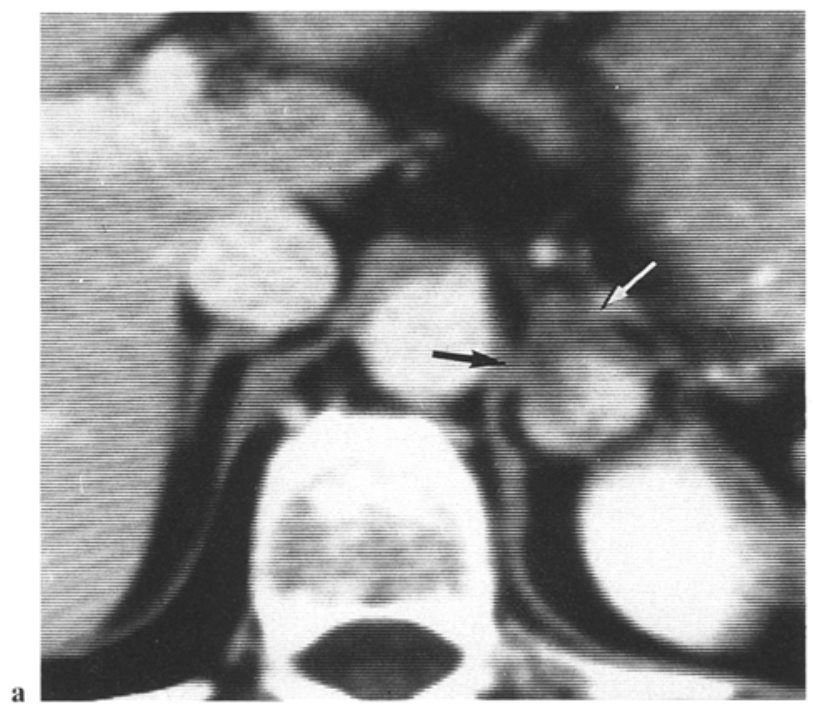

Fig.4a-e. Metastasis of the left adrenal. a CT after contrast medium injection shows a high density lesion with a necrotic component (arrows). MRI b pre and after $1 \mathrm{~min}$ c, 5 min d, $10 \mathrm{~min}$ e Gd-DTPA

with SL/SF values ranging between 1.1 and 1.4 and therefore lower than metastases. In all cases a decreased enhancement was observed with values ranging between 0.7 and 0.8 after $10 \mathrm{~min}$ (Fig. 2).

There is a SL/SF cut-off point between metastases and adenomas between 1.1 and 1.2 where, in later scans (after $10 \mathrm{~min}$ ), all metastases showed higher $\mathrm{SL} / \mathrm{SF}$ values and all benign lesions lower values (Table 1 ).

With GE sequences, it was possible to correctly characterize two lesions, one adenoma (Fig. 3) and one metastasis (Fig. 4), that were equivocal with SE sequences, and to better define a third lesion, interpreted as "possible" metastasis with SE sequences. Only in one case, which was found to be an adenoma at biopsy, did we fail to make a correct diagnosis with either SE or GE sequences.

In one patient with breast cancer, both SE and GE sequences failed to characterize a round homogeneous mass in the right adrenal. On SE T1 sequences, the lesion was isointense with the liver, whereas the signal was partially hyperintense on $\mathrm{T} 2$; the dynamic study showed an adenoma-like enhancement, with a small hyperintense central area in later scans. At biopsy, it was found it to be an adenoma (Table 2).

\section{Discussion}

MR imaging of adrenal glands with SE T1- and T2weighted sequences offers the possibility of differentiating between benign and malignant lesions. On T2weighted images, high signal intensities are suggestive of malignant lesions $[5-8,13,14]$.

By the way there is a large number of indeterminate findings (20-30\%) reported in different series of adrenal lesions, with a typical signal intensity of metastasis or adenoma that were not such at biopsy $[5,15]$.

The aim of our study was a prospective evaluation of "conventional" SE sequences versus dynamic contrast- enhanced images. With dynamic GE sequences the MR criteria for differential diagnosis is the different enhancement characteristic of benign and malignant lesions: according to the literature the former exhibit a mild enhancement and a quick washout, whereas the latter show a more pronounced enhancement and a much slower washout [10-12].

In our series the most consistent results were obtained by comparing the relative SL/SF signal intensities in scans taken after 10 min: metastases have always showed, with respect to adenomas, a much higher SL/SF ratio. We were therefore able to correctly characterize as metastasis a mass with a low SL/SF ratio in early scans, and to recognize as an adenoma a lesion with a high SL/SF ratio after $2 \mathrm{~min}$, in a patient with Cushing's syndrome. The dynamic study after administration of Gd-DTPA was in this case particularly helpful, since the lesion showed a high signal intensity on SE T2-weighted sequences. In comparison with SE T1- and T2-weighted sequences, GE with GdDTPA enhanced dynamic study proved to be more specific since we were able to correctly characterize 18 lesions out of 19 , with an overall diagnostic accuracy of $95 \%$. SE sequences provided useful data for the exact classification of lesions in 16 cases out of 19 , with an overall accuracy of $84 \%$. These data are in agreement with those of another series [11] which studied 38 cases including primary tumors and pheochromocytomas and observed a much

Table 2. Comparison between MR SE sequences and GE sequences with contrast-enhanced dynamic study in the diagnosis of 19 adrenal glands lesions

\begin{tabular}{llll}
\hline & \multicolumn{3}{l}{ Correct Diagnosis } \\
\cline { 2 - 4 } & Cases & MR SE & MR GE Sequences \\
& $(n=19)$ & Sequences & + Gd-DTPA \\
\hline Adenomas & 10 & 8 & 9 \\
Metastases & 9 & 8 & 9 \\
\hline
\end{tabular}


higher diagnostic accuracy in GE sequences with GdDTPA versus SE sequences in the differential diagnosis of benign and malignant lesion.

In conclusion, this study reveals that MR, performed with a Gd-DTPA enhanced dynamic study, allows the characterization of adenomas and metastases with a greater accuracy compared with that of "conventional" SE sequences. We therefore believe that GE MR imaging with Gd-DTPA enhanced dynamic studies should be performed in all cases of equivocal adrenal masses.

\section{References}

1. Commons RA, Callaway CP (1984) Adenomas of the adrenal cortex. Arch Intern Med 8: 37-41; 1984

2. Lee MJ, Hahn PF, Papanicolaou N, Egglin TK, Saini S, Mueller PR, Simeone JF (1991) Benign and malignant adrenal masses: CT distinction with attenuation coefficients, size and observer analysis. Radiology 179: 415-418

3. Miyake H, Maeda H, Tashiro M (1989) CT of adrenal tumors: frequency and clinical significance of low attenuation lesions. AJR 152: 1005-1007

4. Liessi G, Spigariol F (1988) Ruolo della TC e degli US nella diagnosi delle neoplasie surrenali a matrice adiposa. Radiol Med 75 : 195-199

5. Baker ME, Blinder R, Spritzer C, Leight GS, Herfkens RJ, Dunnick NR (1989) MR evaluation of adrenal masses at 1.5 T. AJR 153: $307-312$
6. Glazer GM, Woolsey EJ, Borrello J, Francis IR, Aisen AM, Bookstein F, Amendola MA, Gross MD, Bree RL, Martel W (1986) Adrenal tissue characterization using MR imaging. Radiology 158: 73-79

7. Kier R, McCharty S (1989) MR characterization of adrenal masses: field strength and pulse sequences considerations. Radiology 17: 671-674

8. Rafal RB, Kosovsky PA, Markisz JA (1990) Magnetic Resonance Imaging of the adrenal glands. A subject review. Clin Imaging 14: 1-10

9. Remer EM, Weinfeld RM, Glazer GM, Quint LE, Francis IR, Gross MD, Bookstein FL (1989) Hyperfunction and nonhyperfunction benign adrenal cortical lesions: characterization and comparison with MR imaging. Radiology 171: 681-685

10. Krestin GP, Friedmann G, Fischback R, Neufang KFR, Allolio B (1991) Evaluation of adrenal masses in oncologic patients: dynamic contrast-enhanced MR vs CT. J Comput Assist Tomogr 15: $104-110$

11. Krestin GP, Steinbrich W, Friedman G (1989) Adrenal masses: evaluation with fast dynamic gradient-echo MR imaging and GdDTPA-enhanced dynamic studies. Radiology 171: 675-680

12. Krestin GP (1990) Dynamic renal and adrenal studies with GdDTPA. In: G. Bydder et al. Contrast Media in MRI, Medicom Europe B. V.: Berlin, pp 327-339

13. Chang A, Glazer HS, Lee JKT, Ling D, Heiken JP (1987) Adrenal gland: MR imaging. Radiology 163: 123-128

14. Glazer GM (1988) MR imaging of the liver, kidneys and adrenal glands. Radiology 166: 303-312

15. Baker ME, Spritzer C, Blinder R, Herfkens RJ, Leight GS, Dunnick NR (1987) Benign adrenal lesions mimicking malignancy on MR imaging: report of two cases. Radiology 163:669-671 